\title{
Controlling ion fluxes during reactive sputter-deposition of $\mathrm{SnO}_{2}: \mathrm{F}$
}

\author{
Timo Jäger, ${ }^{1, a)}$ Yaroslav E. Romanyuk, ${ }^{1}$ Ayodhya N. Tiwari, ${ }^{1}$ and André Anders ${ }^{2}$ \\ ${ }^{1}$ Empa-Swiss Federal Laboratories for Materials Science and Technology, Laboratory for Thin Films and \\ Photovoltaics, Überlandstrasse 129, 8600 Dübendorf, Switzerland \\ ${ }^{2}$ Lawrence Berkeley National Laboratory, 1 Cyclotron Road, Berkeley, California 94720, USA
}

(Received 9 April 2014; accepted 24 June 2014; published online 16 July 2014)

\begin{abstract}
Magnetron sputtering of fluorine-doped tin oxide (FTO) is a scalable deposition method for largearea transparent conducting films used in fenestration, photovoltaics, and other applications. The electrical conductivity of sputtered FTO is, however, lower than that of spray-pyrolized FTO because of the ion damage induced by high energy ions leading to a reduction of the crystal quality in sputtered FTO films. In this study, various ion species present during the reactive sputtering of a metallic tin target in a mixed $\mathrm{Ar} / \mathrm{O}_{2} / \mathrm{CF}_{4}$ atmosphere are systematically characterized by energy and mass spectrometry, and possible ways of controlling the ion fluxes are explored. Ion energy distribution functions (IEDFs) of the negative ions $\mathrm{F}^{-}$and $\mathrm{O}^{-}$exhibit large peaks at an energy corresponding to the full target voltage. Although the applied partial pressure of $\mathrm{CF}_{4}$ is about $1 / 30$ than that of $\mathrm{O}_{2}$, the obtained IEDFs of $\mathrm{F}^{-}$and $\mathrm{O}^{-}$have comparable peak height, which can be attributed to a higher electronegativity of $\mathrm{F}$. The IEDFs of positively charged $\mathrm{O}^{+}, \mathrm{O}_{2}{ }^{+}, \mathrm{Ar}^{+}$, and $\mathrm{Sn}^{+}$species have their peaks around $2-8 \mathrm{eV}$. To control ion fluxes a solenoid or permanent magnets were placed between the target and the mass spectrometer. The flux of positive ions could be varied by several orders of magnitude as a function of the applied current through the solenoid, whereas the high-energy $(>100 \mathrm{eV})$ negative $\mathrm{F}^{-}$and $\mathrm{O}^{-}$ions were not notably deflected. By using permanent magnets with the $\mathbf{B}$-field orthogonal to the ion trajectory, the flux of $\mathrm{O}^{-}$ions could be decreased by two orders and the exposure to the high-energy $\mathrm{F}^{-}$ions was completely suppressed. (C) 2014 AIP Publishing LLC. [http://dx.doi.org/10.1063/1.4887119]
\end{abstract}

\section{INTRODUCTION}

The microstructure of a sputter-deposited thin film is influenced by several parameters, such as the substrate temperature, total pressure, and partial pressure of the reactive gas as described by the zone structure model of Thornton. ${ }^{1}$ In addition, the effect of the ion flux $J_{\mathrm{i}}$ and ion energy $E_{\mathrm{i}}$ on the morphology of the growing film was also investigated, and the zone model has been extended accordingly.,3 The ion flux and energy are particularly critical for obtaining high-quality layers of transparent conducting oxides (TCOs) that combine high electrical conductivity and visible transparency. Apart from some amorphous transparent conducting oxides used for transparent electronics, ${ }^{4,5}$ both film properties generally require a high crystalline quality, ${ }^{6,7}$ and therefore bulk displacements by ion bombardment with energy $>100 \mathrm{eV}$ during film growth should be avoided. In contrast, the low energy ions $(\leq 20 \mathrm{eV})$ that influence only the first few monolayers can be beneficial for increasing the adatom mobility and removing weakly bonded surface atoms.

The ion energy $E_{\mathrm{i}}$ in direct current (DC) sputtering is usually determined by the highest potential in the system, which is typically the target voltage of several hundred V. In this case, ions are created near the target surface and accelerated by the full plasma sheath voltage. The role of these high energy ions in sputter-deposited TCOs has been reviewed by Ellmer and Welzel, ${ }^{8}$ who investigated negative oxygen ions in sputtering of various metals, such as In, InSn, Ti, Zn, and

\footnotetext{
a) Author to whom correspondence should be addressed. Electronic mail: timo.jaeger@empa.ch.
}

Sn. It was noted that sputtering $\mathrm{Sn}$ in $\mathrm{O}_{2}$ atmosphere yields the highest flux of negative $\mathrm{O}^{-}$ions, which was proposed to be a possible reason for a rather low electrical conductivity limited to $<1000 \mathrm{~S} / \mathrm{m}$ for sputter-deposited $\mathrm{SnO}_{2}$ doped with $\mathrm{F}, \mathrm{Sb}$, or Mo. ${ }^{6,9}$ In order to investigate the detrimental highenergy ions, ion energy distribution functions (IEDF) should be measured during magnetron sputtering, as it has been demonstrated for the reactive sputtering of $\mathrm{Zn}, \mathrm{ZnO}: \mathrm{Al}$, and $\mathrm{Al}^{10-12} \mathrm{~A}$ recent study suggested that high transversal electrical fields between target and substrate using a plasma lens could effectively tune the high energy ion fluxes and lead to an improvement in film quality for $\mathrm{ZnO}: \mathrm{Al} .{ }^{13}$ Here, we follow up on this concept and present possible ways to control the ion flux during reactive sputter-deposition of $\mathrm{SnO}_{2}: \mathrm{F}$ (fluorine-doped tin oxide (FTO)) and characterize the resulting IEDFs of various ion species present in the plasma. In the first approach of the "plasma lens," a solenoid is placed between the target and the substrate position whereby a current applied through the solenoid creates a magnetic field necessary to guide electrons, which in turn setup an electric field that affects the trajectories of the ions. In a second approach with the "plasma trap," the deflection of ions is achieved by a strong transversal magnetic field, which affects the ion trajectories directly as well as indirectly, namely, via the electric field set up by the magnetized, trapped electrons.

\section{EXPERIMENTAL DETAILS}

The high vacuum system was equipped with mass flow controllers for multiple gas injection, residual gas analyzer, turbomolecular and cryogenic pumps. Planar magnetrons 
TABLE I. Sputter parameters of the configurations plasma lens and plasma trap

\begin{tabular}{lcc}
\hline \hline Sputter parameters/approach & Plasma lens & Plasma trap \\
\hline Sputter power & $100 \mathrm{~W}$ & $157 \mathrm{~W}$ \\
Target voltage & $263 \mathrm{~V}$ & $272-311 \mathrm{~V}$ \\
Target current & $0.38 \mathrm{~A}$ & $0.58-0.51 \mathrm{~A}$ \\
Pumping mode & Cryogenic & Cryogenic + turbomolecular \\
Total pressure & $0.5 \mathrm{~Pa}$ & $0.2-0.3 \mathrm{~Pa}$ \\
Ar flow & $19.4 \mathrm{sccm}$ & $14.9 \mathrm{sccm}$ \\
$\mathrm{O}_{2}$ flow & $17.3 \mathrm{sccm}$ & $9.2-16.4 \mathrm{sccm}$ \\
$\mathrm{CF}_{4}$ partial pressure & $0-0.01 \mathrm{~Pa}$ & $0.003 \mathrm{~Pa}$ \\
Target-to-EQP300 distance & $56 \mathrm{~cm}$ & $38 \mathrm{~cm}$ \\
Distance target to plasma lens/trap & $4.5 \mathrm{~cm}$ & $7 \mathrm{~cm}$ \\
Distance Plasma lens/trap to & $15 \mathrm{~cm}$ & $19 \mathrm{~cm}$ \\
EQP300 (orifice) & & \\
\hline
\end{tabular}

with a diameter of $7.62 \mathrm{~cm}$ (3 in.) were used in an unbalanced configuration. Metallic tin targets (99.999\%) were sputtered in a mixed $\mathrm{Ar} / \mathrm{O}_{2} / \mathrm{CF}_{4}$ atmosphere with sputter parameters described in Table $\mathrm{I}$.

Two approaches were explored to control the ion flux in DC magnetron sputtering:

(i) Plasma lens: A solenoid was placed between the target and the substrate creating an axial magnetic field $B$ by applying a direct current $I$ up to $337 \mathrm{~A}$. The solenoid made of $\mathrm{Cu}$ tubing was water-cooled and had a diameter of $12 \mathrm{~cm}$, length of $36 \mathrm{~cm}$, and 28 windings, yielding a maximum $B$ of $33 \mathrm{mT}$.

(ii) Plasma trap: Permanent rare-earth magnets mounted on an Al frame were placed between the target and the spectrometer in the configuration depicted in Fig. 3(a). The opposite poles were connected with a U-shaped soft iron construction to enhance the field strength inside and to decrease stray fields outside the magnet array, which could disturb the ion trajectories inside the spectrometer. The maximal field strength $B$ inside the plasma trap was $200 \mathrm{mT}$ corresponding to an expected gyration radius of about $0.1 \mathrm{~m}$ for oxygen ions and about $0.6 \mathrm{~mm}$ for electrons.

The plasma diagnostics were performed with an EQP300 mass and energy analyzer (Hiden Analytical) yielding IEDFs. The EQP300 instrument with a $100 \mu \mathrm{m}$ entrance orifice was aligned on axis with the target center and orthogonal to the target surface. The detector of this instrument recorded counts per second as a function of the discrimination voltage for a specific mass to charge ratio $m / Q$. The energetic resolution of the EQP300 instrument was 0.5 and $0.1 \mathrm{eV}$ for measuring IEDFs of negative and positive ions, respectively. The population of multiply-charged ions in DC magnetron sputtering is low ${ }^{14}$ and therefore the discrimination voltage of the spectrometer was directly converted into energy.

\section{RESULTS AND DISCUSSION}

\section{A. Plasma lens}

First, the possibility to control ion fluxes with a solenoid is investigated. Fig. 1(a) exhibits photographs of the plasma near the target surface for applied DC coil currents $I$ of 0,58 , 138, and $337 \mathrm{~A}$. The magnetic field created by the solenoid leads to an increased unbalancing of the magnetron and the plasma can escape from the target region at an enhanced rate. The escaped plasma is then guided through the solenoid with a narrowing channel for increasing solenoid current. In order to estimate the electric force acting on different ion species, the floating potential across the plasma lens was measured with a Langmuir probe (Fig. 1(b)). The probe was constructed of a $\sim 1 \mathrm{~mm}$ diameter tungsten wire encapsulated in a ceramic pipe and a glass pipe to prevent shorting to ground potential. The tungsten probe exposed to the plasma had a length of $\sim 2 \mathrm{~mm}$. The floating potential ${ }^{15}$

$$
V_{\mathrm{f}}=V_{\mathrm{p}}+\left(\frac{k T_{\mathrm{e}}}{e}\right) \ln \left(0.6 \sqrt{\frac{2 \pi m_{\mathrm{e}}}{m_{\mathrm{i}}}}\right)
$$

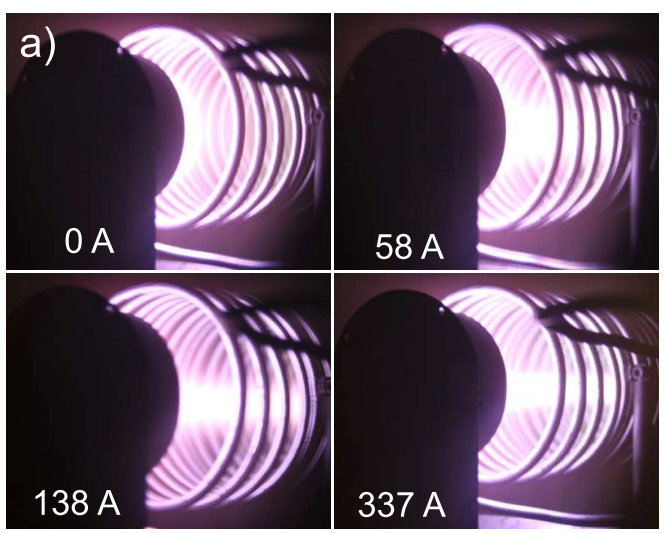

b)

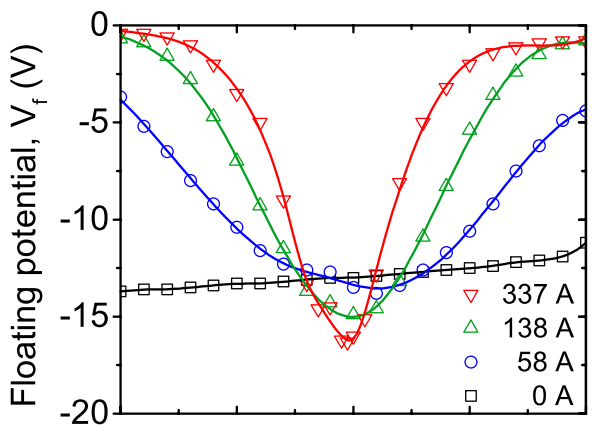

C)

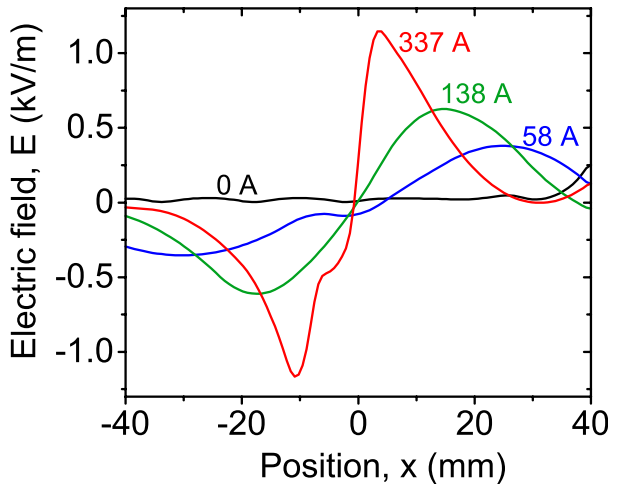

FIG. 1. (a) Pictures of plasma lens at an applied current of $0,58,138$, and $337 \mathrm{~A}$ during sputtering of $\mathrm{Sn}$ in an $\mathrm{Ar} / \mathrm{O}_{2} / \mathrm{CF}_{4}$ atmosphere: The magnetron is to the left and the plasma enters the solenoid primarily on axis. (b) Floating potential $V_{\mathrm{f}}$ measured across the plasma lens at a distance of $\sim 25 \mathrm{~cm}$ from the target for different currents $I$ shown with spline interpolations and (c) calculated electric field $E$. 
was measured for coil currents $I$ of $0,58,138$, and $337 \mathrm{~A}$ as a function of the distance $x$ from the lens center and is plotted in Fig. 1(b). $V_{\mathrm{p}}$ is the plasma potential, $k$ is the Boltzmann constant, $T_{\mathrm{e}}$ is the electron temperature, $e$ is the elementary charge, $m_{\mathrm{i}}$ and $m_{\mathrm{e}}$ are the ion and electron mass, respectively. When no current is applied, $V_{\mathrm{f}}$ is almost constant across the solenoid because the plasma is confined to the target by the magnetron at a distance of $\sim 25 \mathrm{~cm}$ away from the Langmuir probe. When the plasma lens is powered, a potential dip is formed along the axis of the solenoid ${ }^{16}$ and the dip depth is increasing for higher DC currents. The width of the potential dip decreases because of a higher $B$-field that becomes visible in the narrowing plasma in Fig. 1(a). The magnetic field lines of the solenoid intersect with the magnetic field of the magnetron creating a potential valley in the center of the solenoid. The magnetic field is strong enough to magnetize electrons (electron gyration radius $\ll$ radius of solenoid), while ions are not magnetized due to their large mass. The combined effect of electron guiding by the magnetic field and ion guiding by the electric field of the potential valley leads to guiding of the plasma through the solenoid. In addition to plasma guiding, the solenoid provides excitation and ionization of neutral and ion species. The potential valley is expected to depend also on the solenoid to target distance as it affects field lines which intersect the negatively biased target. ${ }^{17}$ The resulting electric field strength was calculated from a spline fit of the floating potential $V_{\mathrm{f}}$ by a differentiation with $E(x)=\partial V_{\mathrm{f}} / \partial x$ in Fig. 1(c) neglecting the term with $\partial T_{\mathrm{e}} / \partial x$ as the electron temperature changes insignificantly. The build-up radial potential distribution is attractive (channeling) for positive ions and expulsive for negative ions. The maximum electric field $|E|$ is calculated to be $\sim 0,0.38,0.63$, and $1.2 \mathrm{kV} / \mathrm{m}$ for $0,58,138$, and $337 \mathrm{~A}$, respectively. The steep increase in $E$ for $x>30 \mathrm{~mm}$ with no current applied can be attributed to a measurement artifact and is not considered in this discussion. The solenoid is therefore a focusing/defocusing lens for positive/negative ions respectively.

The IEDFs of the negative ions $\mathrm{O}^{-}$and $\mathrm{F}^{-}$detected at a target voltage of $263 \mathrm{~V}$ and a $\mathrm{CF}_{4}$ partial pressure $p p\left(\mathrm{CF}_{4}\right)$ of $0.01 \mathrm{~Pa}$ are plotted in Fig. 2(a). IEDFs of both ion species have pronounced peaks at $290 \mathrm{eV}$, which is somewhat higher than the full discharge voltage of $263 \mathrm{~V}$. IEDFs of negative ions with energies $>50 \mathrm{eV}$ are independent of the plasma lens current $I$, indicating that the electric field induced by the plasma lens is not sufficient to deflect high energy ions. Fig. 1(c) shows that high enough electric fields can be obtained only $1-2 \mathrm{~cm}$ away from the center of the solenoid lens, and that in the very center of the solenoid $E$ is close to zero as depicted in Fig. 1(c). As a result, the total deflection length of the plasma lens is not enough to suppress the substrate exposure to the high-energy $\mathrm{O}^{-}$and $\mathrm{F}^{-}$.

For both negative ions, $\mathrm{O}^{-}$and $\mathrm{F}^{-}$, there is an increase in IEDF towards low energy, which can be attributed to the ions accelerated in the plasma by only a fraction of the target voltage or those undergoing inelastic scattering. It has to be noted that the acceptance angle of the mass and energy analyzer is dependent on the ion energy. Namely, for the EQP300 instrument, the acceptance angle is $<3^{\circ}$ for ion energies $>30 \mathrm{eV}$ but it increases substantially to over $20^{\circ}$ when low energy $(<1 \mathrm{eV})$ ions are measured. ${ }^{18-20}$ Therefore, the strong peak at low ion energies is inflated.

Although the partial pressure of $\mathrm{CF}_{4}$ is about 20 times less than $p p\left(\mathrm{O}_{2}\right)$, the absolute count rate for $\mathrm{F}^{-}$is 10 times a) Negative ions

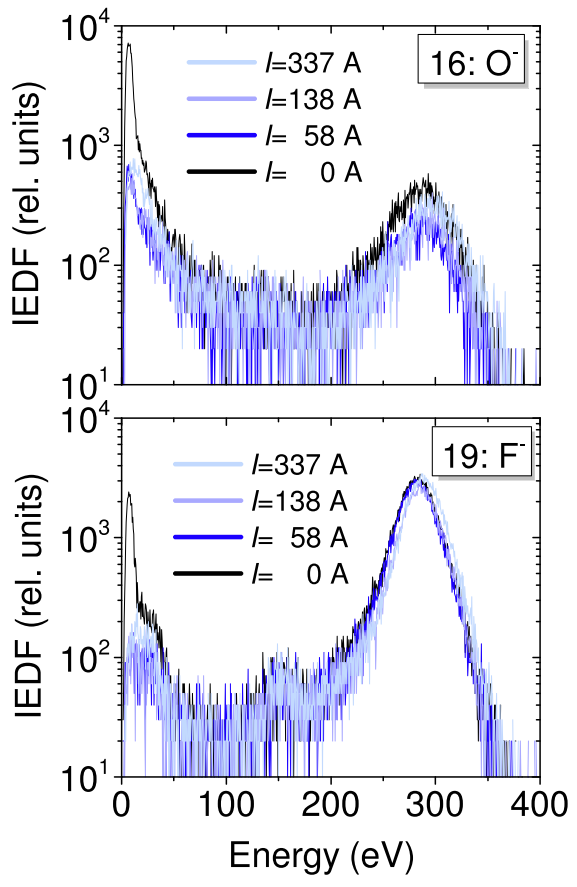

b) Positive ions

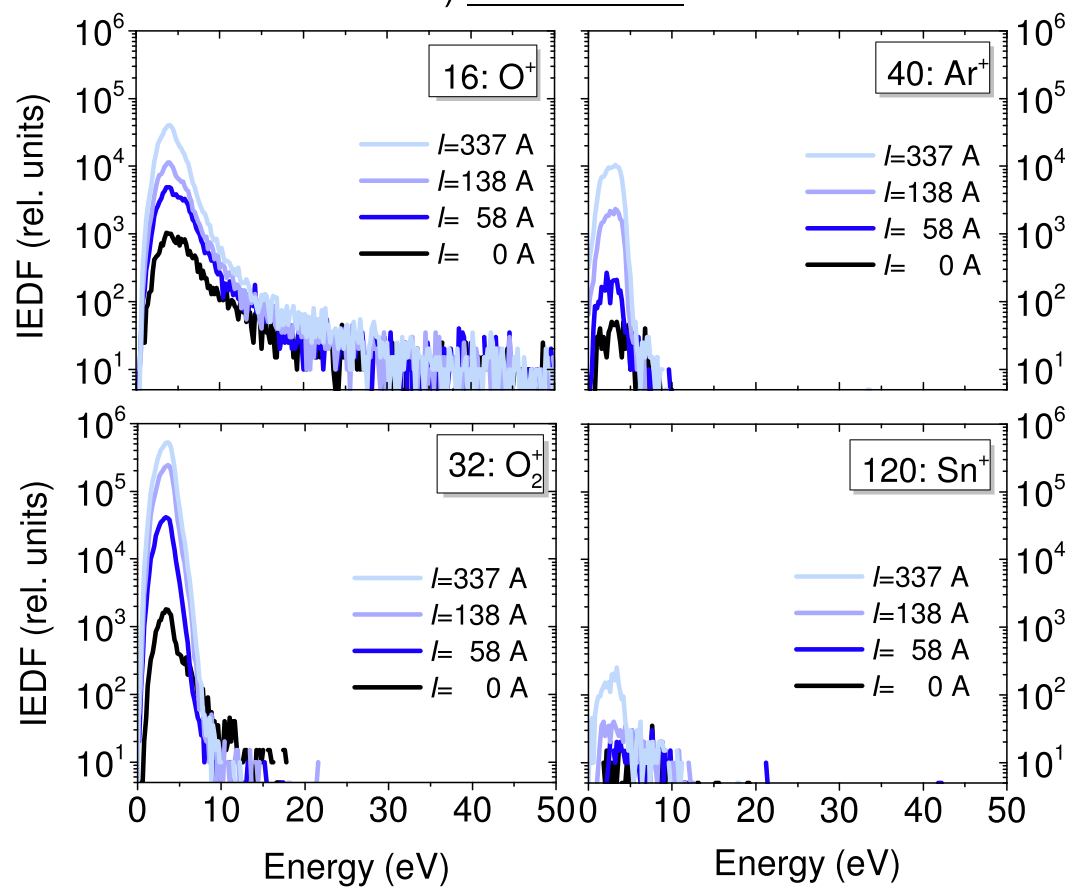

FIG. 2. IEDFs of (a) negative ions and (b) positive ions for applied plasma lens current $I$ of $0,58,138$, and 337 A during sputtering of Sn in an Ar/O $2 / \mathrm{CF}_{4}$ atmosphere. The discrimination voltage of the EQP300 mass and energy analyzer was directly converted into a energy since we know we deal mainly with singly charged particles. The signal measured in cps is shown as the IEDF in relative units. 
larger than for $\mathrm{O}^{-}$at $290 \mathrm{eV}$. Since the bond-dissociation energies $D_{0}$ are similar $\left(D_{0}\right.$ of $\mathrm{O}_{2} \rightarrow 2 \mathrm{O}$ is $498.4 \mathrm{~kJ} / \mathrm{mol}$ and $D_{0}$ of $\mathrm{CF}_{4} \rightarrow \mathrm{CF}_{3}+\mathrm{F}$ is $\left.506 \mathrm{~kJ} / \mathrm{mol}\right){ }^{21}$ the reason for the larger $\mathrm{F}^{-}$peak is the higher electronegativity $\chi$ of $\mathrm{F}$ compared with $\mathrm{O}\left(\chi_{\mathrm{F}}=3.98\right.$ and $\chi_{\mathrm{O}}=3.44$ in the Pauling scale), ${ }^{22}$ which results in higher concentration of $\mathrm{F}^{-}$even though the $\mathrm{CF}_{4}$ partial pressure is lower than $p p\left(\mathrm{O}_{2}\right)$. Additionally, when sputtering with $\mathrm{CF}_{4}$, part of the oxygen is bound into $\mathrm{CO}$ and $\mathrm{CO}_{2}$ that were measured with the Hiden spectrometer in the residual gas analyzer mode.

The influence of the plasma lens is much more dramatic for the positive low-energy ions. Fig. 2(b) shows that the IEDFs of positive ions are tunable by several orders of magnitude using a plasma lens where the integrated count rate increases roughly linearly with the coil current $I$. IEDFs were measured at zero $\mathrm{CF}_{4}$ flow for $m / Q=16,32,40$, and 120 corresponding to the $\mathrm{O}^{+}, \mathrm{O}_{2}{ }^{+}, \mathrm{Ar}^{+}$, and $\mathrm{Sn}^{+}$ion species, respectively (Fig. 2(b)). Two mechanisms contribute to the enhanced ion count. First, the plasma lens provides excitation and ionization of neutral species, thereby increasing the total ion to neutral ratio $J_{\mathrm{i}} / J_{\mathrm{n}}$. Furthermore, the build-up of electric field inside the solenoid guides the positive ions along the lens axis yielding a higher ion flux at the detector.

All measured IEDFs show a maximum at a low energy of $2-5 \mathrm{eV}$. A high energy tail up to $30 \mathrm{eV}$ is visible for $\mathrm{O}^{+}$, and for ion energies $>30 \mathrm{eV}$, the signal is low $<40 \mathrm{cps}$ (counts per second) with a noise background that is not much lower. Most sputtered atoms are in the range from 1 to $10 \mathrm{eV}$, with the maximum of the energy distribution corresponding to half the sublimation energy. ${ }^{23}$ These neutral target atoms are ionized in the plasma and can be additionally accelerated by the sheath just outside the EQP300. The ion energy is therefore limited to several tens of $\mathrm{eV}$ which is
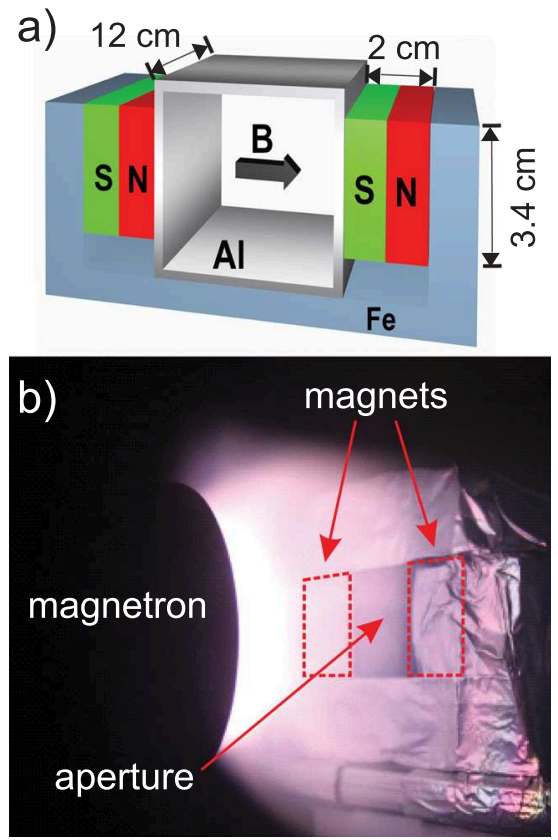

FIG. 3. (a) Schematic of the plasma trap depicted without the Al foil protection. (b) Photograph of sputtering of $\mathrm{Sn}$ in an $\mathrm{Ar} / \mathrm{O}_{2} / \mathrm{CF}_{4}$ atmosphere using a plasma trap with an aperture of $4 \times 4 \mathrm{~cm}^{2}$. The magnet arrays are marked with a dashed frame. consistent with our measurements, where the energies of positive ions do not exceed $50 \mathrm{eV}$ and have a sharp maximum at $2-5 \mathrm{eV}$. It is worth mentioning that the increased ionization of oxygen atoms leads to transparent $\mathrm{SnO}_{2}: \mathrm{F}$ films which can be deposited at much lower $\mathrm{O}_{2}$ partial pressure while still sputtering $\mathrm{Sn}$ in the metallic mode. The transition region, characterized by a sharp drop of the target voltage with increasing oxygen partial pressure, occurs in the region of a relative $\mathrm{O}_{2} / \mathrm{Ar}+\mathrm{O}_{2}$ flow between 40 and $60 \%$ in this sputtering setup. The plasma lens enabled the deposition of transparent, although not conductive, $\mathrm{SnO}_{2}: \mathrm{F}$ films with a relative $\mathrm{O}_{2}$ flow of only $17 \%$ at an applied current $I$ of $138 \mathrm{~A}$, a sputter power of $157 \mathrm{~W}$ (nominal power density of $3.4 \mathrm{~W} / \mathrm{cm}^{2}$ ), and a total pressure of $20 \mathrm{mPa}$. The resulting deposition rate was $\sim 3.3 \mathrm{~nm} / \mathrm{min}$ in a region with a radius of $2 \mathrm{~cm}$ around the center. Outside this region, the film was metallic and clearly distinct from the area inside where oxygen was activated by the guided plasma.

A technical implementation is mainly limited because of the reduced deposition rate compared with conventional DC sputter deposition, due to a rather large target-to-substrate distance. FTO films by conventional reactive sputtering yielded a deposition rate of $21 \mathrm{~nm} / \mathrm{min}$ at a total pressure of $0.2 \mathrm{~Pa}$ and a shorter target-to-substrate distance of $20 \mathrm{~cm}$. Only part of the rate loss can be recovered by the increased ionization and guiding of sputtered atoms (now ionized) by the plasma lens. This indicates that a plasma lens approach may be useful for high-power impulse magnetron sputtering (HiPIMS) with substantial ionization but not for conventional reactive sputtering.

\section{B. Plasma trap}

In order to suppress high-energy negative ions, we applied a strong magnetic field perpendicular to the targetto-substrate direction. SmCo magnets with fields up to $200 \mathrm{mT}$ (measured in the center between opposing magnets) were placed between the target and the spectrometer in the configuration depicted in Fig. 3(a). The magnet array was protected from the plasma by $\mathrm{Al}$ foil with an aperture of $4 \times 4 \mathrm{~cm}^{2}$ visible in Fig. 3(b). Magnetic field lines parallel to the target prevent the plasma from penetrating the aperture and therefore effectively act as a plasma trap. Furthermore, the plasma near the target is affected by the plasma trap and is pushed towards the direction of the $\mathbf{B}$-field of the trap.

The sputter yield and ion energy distributions are changing with target poisoning leading to FTO films with optoelectrical properties corresponding to the surface state of the target. Therefore, the IEDF of $\mathrm{O}^{-}$was investigated for different target poisoning states, particularly as the magnetic field near the target is affected by the $\mathbf{B}$-field of the plasma trap. The target poisoning is shown in Fig. 4(a) for the deposition with and without additional magnets. The target voltage for all applied $\mathrm{O}_{2}$ flows remains almost unchanged with the installation of the plasma trap (Fig. 4(a)) implying that the ion energy corresponding to the target voltage is the same for both deposition modes.

There are two regions that affect the ion formation and ion flux: the target surface, and the plasma transport section. 
As the $\mathrm{O}_{2}$ flow is increased, the target surface is poisoned changing the chemical surface composition. As a consequence, the deposition rate is decreased and the ion flux may also change significantly. Additionally, the scattering mechanisms in the plasma change with increasing oxygen partial pressure and different ion species present. Fig. 4(b) shows IEDFs of $\mathrm{O}^{-}$measured at three different working points, namely, in the transition region (9.2 and $13.3 \mathrm{sccm} \mathrm{O}_{2}$ ) and in the poisoned mode $\left(16.4 \mathrm{sccm} \mathrm{O}_{2}\right)$ without the plasma trap. The main peak shifts with decreasing target voltage during poisoning from $285 \mathrm{eV}$ at $9.2 \mathrm{sccm} \mathrm{O}_{2}$ towards lower energies of 259 and $233 \mathrm{eV}$ at 13.3 and $16.4 \mathrm{sccm} \mathrm{O}_{2}$, respectively. This indicates the origin of the energy gain of $\mathrm{O}^{-}$to be near the target surface and the direct conversion of the target voltage to the most probable ion energy. The peak intensity decreases with target poisoning which is due to a lower sputter yield of the poisoned target. Both, enhanced scattering at higher total pressure and different ion formation at the target can lead to energy peak broadening which is apparent for increasing $\mathrm{O}_{2}$ flow.

The effect of the plasma trap on the IEDFs is shown in Fig. 5 at a target voltage of $\sim 300 \mathrm{~V}$ and an $\mathrm{O}_{2}$ flow of 9.2 sccm. For the conventional deposition mode, the IEDFs of $\mathrm{O}^{-}$and $\mathrm{F}^{-}$show pronounced peaks at $\sim 280 \mathrm{eV}$ reflecting the target potential of $300 \mathrm{~V}$ (Fig. 5(a)). With the plasma

a)

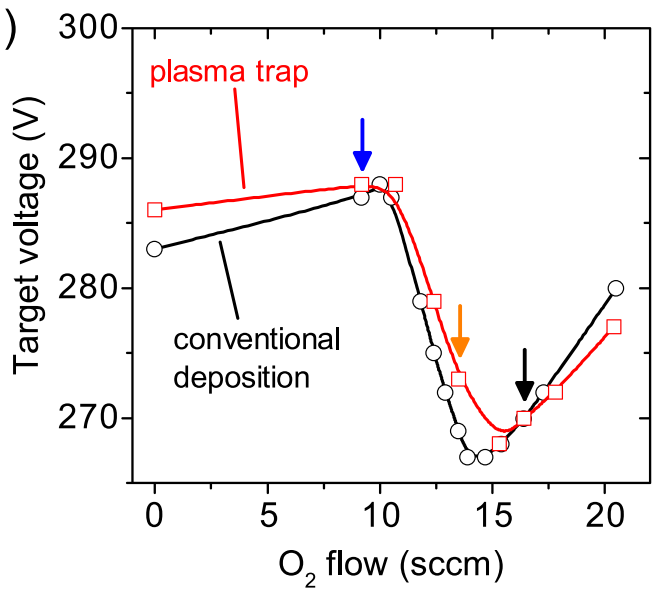

b)

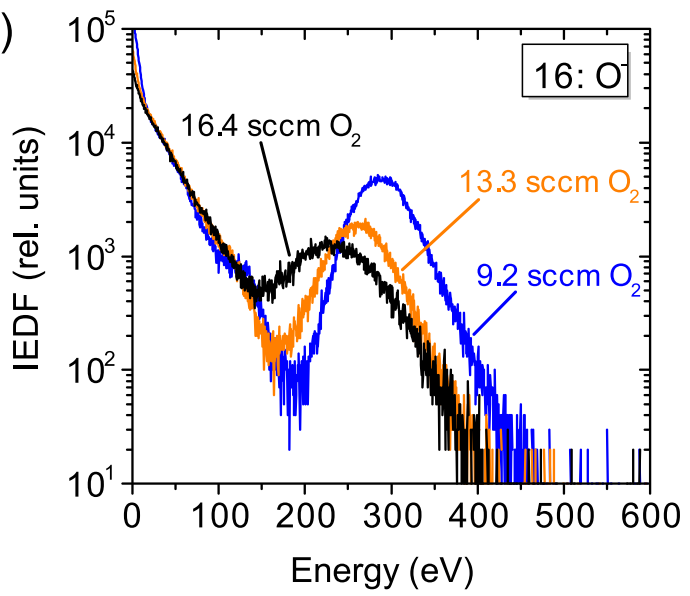

FIG. 4. (a) Target poisoning: the target voltage as a function of the $\mathrm{O}_{2}$ flow with and without the plasma trap. (b) IEDF of $\mathrm{O}^{-}$for different flows of $\mathrm{O}_{2}$ without the plasma trap. The target voltage corresponding to each $\mathrm{O}_{2}$ flow is labeled in (a) with a correspondingly colored arrow. trap, the flux of negative ions decreases by at least two orders of magnitude and the high energy bombardment is successfully suppressed. The acceptance angle of the energy and mass spectrometry is smaller than $5^{\circ}$ for ion energies larger than $10 \mathrm{eV},{ }^{20}$ and therefore the suppression of high energy ions can be attributed to the effect of the magnet array and the geometric influence of the trap aperture can be neglected. The ion flux of $\mathrm{F}^{-}$vanishes completely for $>200 \mathrm{eV}$ while there is still a peak observable for $\mathrm{O}^{-}$. We assume that the residual signal of $\mathrm{O}^{-}$comes from oxygen ions travelling beyond the plasma trap. Since this path will be retarded by scattering, the $\mathrm{F}^{-}$signal is much weaker because of a lower $\mathrm{CF}_{4}$ partial pressure. This means that a transversal magnetic field of $200 \mathrm{mT}$ is sufficient to prevent the growing film from ion bombardment.

For the conventional deposition mode, an additional peak at the energy of $125 \mathrm{eV}$ corresponding to roughly half of the target voltage is clearly visible for $\mathrm{F}^{-}$, while for $\mathrm{O}^{-}$, it is apparent as a distinct shoulder. Several processes are possible which could lead to the peak of $\mathrm{F}^{-}$at $125 \mathrm{eV}: X \mathrm{~F}+e^{-}$ $\rightarrow X \mathrm{~F}^{-}$accelerated by the target potential $\rightarrow$ dissociation $\rightarrow X$ $+\mathrm{F}^{-}$, where $\mathrm{X}$ is a $\mathrm{C}, \mathrm{O}$, or $\mathrm{F}$ atom. The molecule $X \mathrm{~F}^{-}$is accelerated to an energy of $\sim 300 \mathrm{eV}$ and after dissociation the energy is distributed between $X$ and $\mathrm{F}^{-}$, where only the fluorine ions are detected by the EQP300 analyzer.

The larger electronegativity of $\mathrm{F}$ is also prominent in this experiment because similar count rates of $\mathrm{F}^{-}$and $\mathrm{O}^{-}$are measured despite the much larger partial pressure of $\mathrm{O}_{2}$ compared with $\mathrm{CF}_{4}$ with $p p\left(\mathrm{O}_{2}\right) \approx 0.1 \mathrm{~Pa}$ and $p p\left(\mathrm{CF}_{4}\right) \approx 0.003 \mathrm{~Pa}$.

However, in contrast to the plasma lens, the plasma trap yields also a reduction in the flux of positive ions (Fig. 5(b)). It is apparent that the plasma trap does not change the shape of the IEDF but only the total intensity. It is remarkable that a quite high flux of positive ions is measured despite the high transversal B-field and the suppression of the plasma inside the trap. As it is very unlikely that positive ions are created after the plasma trap, the measured flux can be attributed to ions which are formed in plasma near the target and undergo multiple scattering until reaching the detector.

\section{Effect of plasma trap on FTO thin films}

The series of FTO films shown in Fig. 6 were deposited in the conventional configuration and using the plasma trap. The target-to-substrate distance of $\sim 20 \mathrm{~cm}$ was kept constant for all depositions modes. The electrical resistivity of both series shows a distinct minimum at an $\mathrm{O}_{2}$ flow of 8.6 and 9.8 sccm for the conventional deposition and the mode with the plasma, respectively. The application of the plasma trap led to a slight improvement in electrical resistivity from $4.9 \times 10^{-3} \Omega \mathrm{cm}$ to $4.0 \times 10^{-3} \Omega \mathrm{cm}$ due to an increase in the carrier density from $5.4 \times 10^{19} \mathrm{~cm}^{-3}$ to $7.1 \times 10^{19} \mathrm{~cm}^{-3}$. The highest electrical mobility of $25 \mathrm{~cm}^{2} / \mathrm{Vs}$ was achieved using the plasma trap. With the plasma trap present, the bombardment of high energy ions is significantly reduced making the film less prone to resputtering and possibly leading to less crystal defects. However, due to the decrease in the low energy ion flux, the working point for optimum film properties is shifted towards larger $\mathrm{O}_{2}$ flows. Therefore, it is 
a) Negative ions
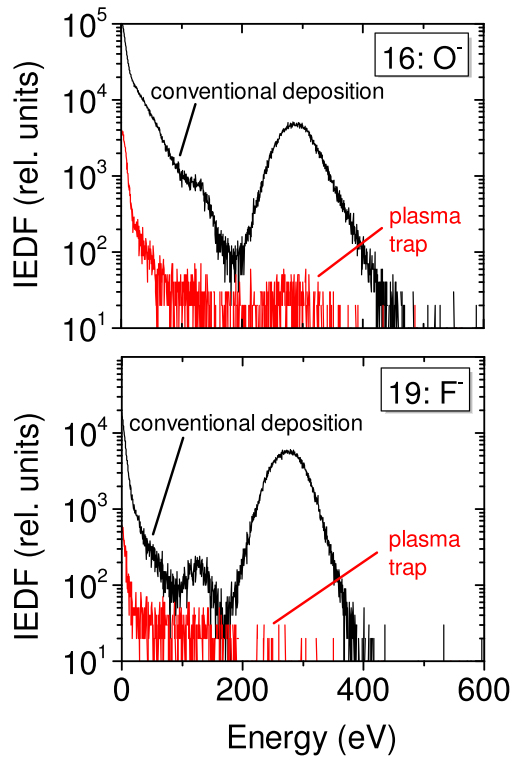

b) Positive ions
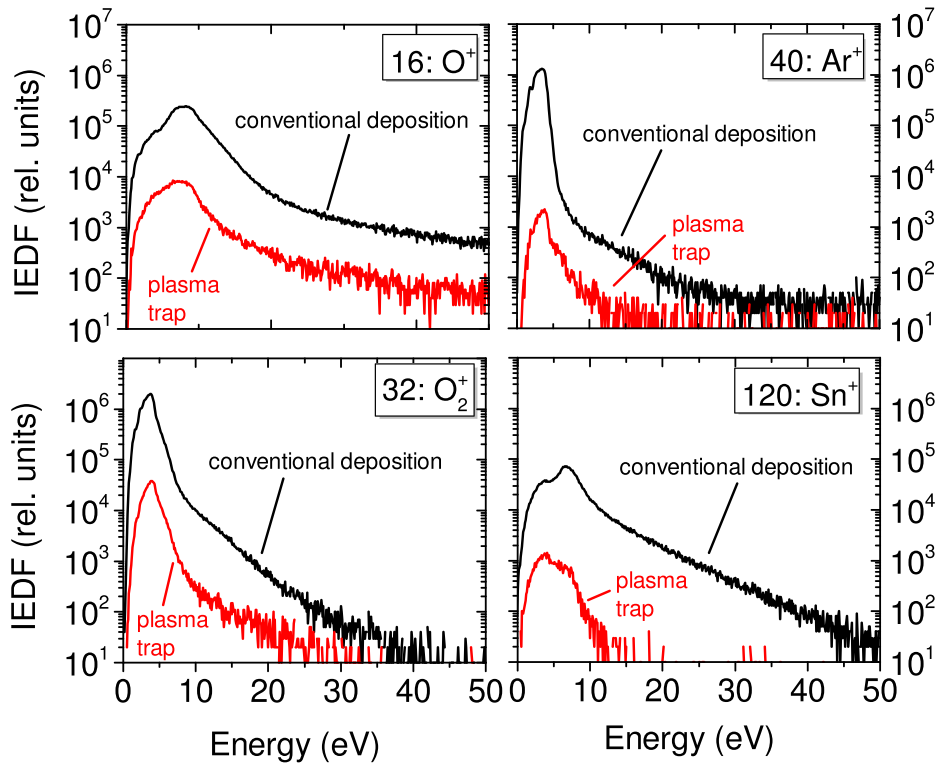

FIG. 5. IEDFs of (a) negative ions and (b) positive ions with (plasma trap) and without (conventional deposition) an additionally installed magnet array during sputtering of $\mathrm{Sn}$ in an $\mathrm{Ar} / \mathrm{O}_{2} / \mathrm{CF}_{4}$ atmosphere. $\mathrm{O}_{2}$ flow was $9.2 \mathrm{sccm}$. The discrimination voltage of the EQP300 mass and energy analyzer was directly converted into a energy since we know we deal mainly with singly charged particles. The signal measured in cps is shown as the IEDF in relative units.

difficult to assign the electrical improvement either to the reduced ion bombardment or to the enhanced dissociation of oxygen due to the strong $\mathbf{B}$-field of the plasma trap.

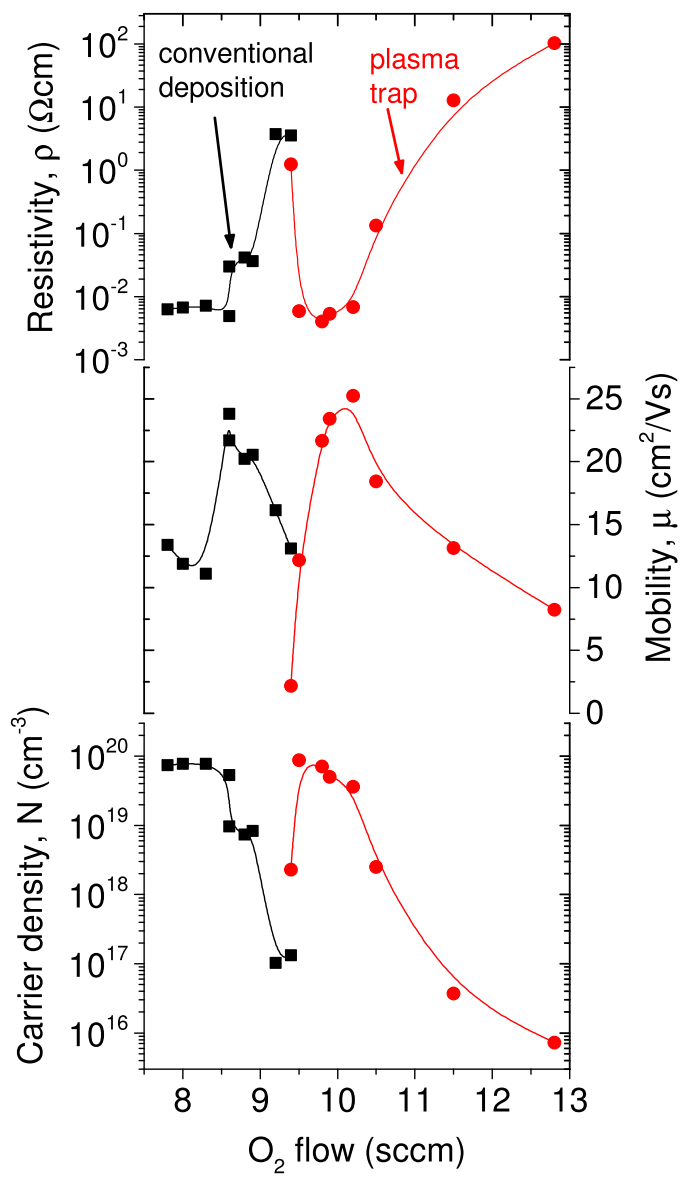

FIG. 6. Electrical resistivity $\rho$, mobility $\mu$, and carrier density $N$ in the conventional sputter configuration (square) and with a plasma trap present (dots). Spline interpolations are for guiding the eye only.
With the installation of the plasma trap, the deposition rate decreased from 21 to $12 \mathrm{~nm} / \mathrm{min}$ mainly caused by the trap aperture and the loss of deflected ions. For a technical application, the aperture can be avoided by using a magnet array with a stronger $\mathbf{B}$-field and thus increasing the distance between the opposing magnets.

\section{SUMMARY AND CONCLUSIONS}

Various ion species present during the reactive sputtering of metallic tin in mixed $\mathrm{Ar} / \mathrm{O}_{2} / \mathrm{CF}_{4}$ atmosphere have been systematically characterized by energy and mass spectrometry. We demonstrate possible ways to control the ion flux in sputter-deposition of $\mathrm{SnO}_{2}: \mathrm{F}$. The flux of the positive ions $\mathrm{O}^{+}, \mathrm{O}_{2}^{+}, \mathrm{Ar}^{+}$, and $\mathrm{Sn}^{+}$can be tuned by several orders of magnitude using the plasma lens formed by a solenoid placed between the target and the substrate position. A DC current is applied to the solenoid leading to an enhanced ionization and guidance of the plasma along the solenoid axis towards the substrate. The flux of positive ions increases almost linearly with the plasma lens current, whereas the flux of high-energy $\mathrm{O}^{-}$and $\mathrm{F}^{-}$accelerated by the full target potential of around $300 \mathrm{~V}$ remains unchanged. Since these high-energy negative ions cause severe damage to the growing film, we applied a strong magnetic field perpendicular to the target-to-substrate direction which effectively acted as a plasma trap for high and low energy ions. Experiments with FTO thin film deposition show certain improvements in electrical properties of FTO films when the plasma trap is present. An additional finding is that because of the large electronegativity of $\mathrm{F}$, the flux of $\mathrm{F}^{-}$is higher than that of $\mathrm{O}^{-}$even at partial pressures of $\mathrm{CF}_{4}$ much lower than $\mathrm{O}_{2}$. In order to reduce the $\mathrm{F}^{-}$ion bombardment of the growing film, one could envisage different strategies, e.g., to decrease the F partial pressure, or to provide the $\mathrm{CF}_{4}$ gas flow directly to 
the substrate surface and thus avoid the target poisoning by fluorine.

\section{ACKNOWLEDGMENTS}

The authors would like to thank Joe Wallig for technical support. Proofreading by Michael Rawlence and art drawing by Bruno Jäger are gratefully acknowledged. The work was partly supported by the Swiss Commission for Technology and Innovation (CTI) under project No. 13708.1 PFFLR-IW. The work at the Lawrence Berkeley National Laboratory was supported by the US Department of Energy under Contract No. DE-AC02-05CH11231.

${ }^{1}$ J. A. Thornton, J. Vac. Sci. Technol. 11, 666 (1974).

${ }^{2}$ I. Petrov, P. B. Barna, L. Hultman, and J. E. Greene, J. Vac. Sci. Technol. A 21, S117 (2003).

${ }^{3}$ A. Anders, Thin Solid Films 518, 4087 (2010).

${ }^{4}$ J. S. Park, W.-J. Maeng, H.-S. Kim, and J.-S. Park, Thin Solid Films 520, 1679 (2012).

${ }^{5}$ A. Walsh, J. L. F. Da Silva, and S.-H. Wei, J. Phys.: Condens. Matter 23, 334210 (2011)

${ }^{6}$ B. Stjerna, E. Olsson, and C. G. Granqvist, J. Appl. Phys. 76, 3797 (1994).
${ }^{7}$ K. Ellmer and R. Mientus, Thin Solid Films 516, 5829 (2008).

${ }^{8}$ K. Ellmer and T. Welzel, J. Mater. Res. 27, 765 (2012).

${ }^{9}$ T. Maruyama and H. Akagi, J. Electrochem. Soc. 143, 283 (1996).

${ }^{10}$ K. Tominaga, Y. Sueyoshi, H. Imai, M. Chong, and Y. Shintani, Jpn. J. Appl. Phys. Part 1 32, 4745 (1993).

${ }^{11}$ T. Welzel, R. Kleinhempel, T. Dunger, and F. Richter, Plasma Process. Polym. 6, S331 (2009).

${ }^{12}$ S. Mraz and J. M. Schneider, Appl. Phys. Lett. 89, 051502 (2006).

${ }^{13}$ A. Anders and J. Brown, IEEE Trans. Plasma Sci. 39, 2528 (2011).

${ }^{14}$ M. Panjan, R. Franz, and A. Anders, Plasma Sources Sci. Technol. 23, 025007 (2014).

${ }^{15}$ R. L. Merlino, Am. J. Phys. 75, 1078 (2007).

${ }^{16}$ E. Byon and A. Anders, J. Appl. Phys. 93, 8890 (2003).

${ }^{17}$ A. Anders, S. Anders, and I. G. Brown, Plasma Sources Sci. Technol. 4, 1 (1995).

${ }^{18}$ E. A. G. Hamers, W. G. J. H. M. van Sark, J. Bezemer, W. J. Goedheer, and W. F. van der Weg, Int. J. Mass Spectrom. Ion Processes 173, 91 (1998).

${ }^{19}$ K. Ellmer, R. Wendt, and K. Wiesemann, Int. J. Mass Spectrom. 223-224, 679-693 (2003).

${ }^{20}$ D. Lundin, P. Larsson, E. Wallin, M. Lattemann, N. Brenning, and U. Helmersson, Plasma Sources Sci. Technol. 17, 035021 (2008).

${ }^{21}$ B. deB Darwent, Bond Dissociation Energies in Simple Molecules (U.S. Department of Commerce, Washington, DC, 1970), p. 20 and 41; available at http://www.nist.gov/data/nsrds/NSRDS-NBS31.pdf.

${ }^{22}$ A. L. Allred, J. Inorg. Nucl. Chem. 17, 215 (1961).

${ }^{23}$ M. W. Thompson, Philos. Mag. 18, 377-414 (1968). 